\title{
Contextual cueing facilitation arises early in the time course of visual search: An investigation with the speed-accuracy tradeoff task
}

\author{
Honami Kobayashi $^{1} \cdot$ Hirokazu Ogawa $^{1}$ \\ Published online: 6 May 2020 \\ (C) The Psychonomic Society, Inc. 2020
}

\begin{abstract}
Visual search is expedited in a spatial context encountered repeatedly. A much-debated question is how early the facilitation by contextual memory arises. The current study examined the possibility of the facilitation of early attentional processing by constructing a descriptive model of the time course of visual search and its facilitation by contextual cueing. Participants in this experiment engaged in a speed-accuracy tradeoff (SAT) task after learning the spatial contexts in a standard visual search task in which they searched for a rotated T target among Ls. In the SAT task, they were required to search for the target and respond immediately when a sound probe was presented, even if they did not find or identify the target when the inter-stimulus interval between the search display and the probe varied from $40 \mathrm{~ms}$ to 2,000 ms. Participants completed two blocks of the SAT task, in which they searched in learned and new contexts. The results of the SAT procedure showed that responses were more accurate in repeated contexts than in new contexts, even when only a brief period of time elapsed after presenting the search display $(>90$ $\mathrm{ms}$ ). We conducted an analysis of the time course of contextual-cueing effects with Bayesian hierarchical modeling, which demonstrated that the rate of increase in accuracy was higher for the repeated than for the new contexts. These findings suggest that early attentional processing is enhanced by learning the contexts, and this enhancement arises very early in the time course of the visual search.
\end{abstract}

Keywords Visual search · Attention and memory · Bayesian modeling

\section{Introduction}

In recent years, there has been an increase in interest in Bayesian statistical modeling in various domains of cognitive psychology, which has led to a proliferation of studies using this quantitative approach (van de Schoot, Winter, Ryan, Zondervan-Zwijnenburg, \& Depaoli, 2017). Bayesian hierarchical modeling, which draws on Bayesian statistical methods, has been used in psychology to describe datageneration processes as probability models, to predict behaviors from the models, and to compare the predictions with observed data. In turn, this allows inference of mechanisms behind the observation through concrete models.

Honami Kobayashi

honami-k@kwansei.ac.jp

1 Department of Psychological Sciences, Kwansei Gakuin University, Hyogo, Japan
Although the number of studies in cognitive psychology employing the Bayesian approach has been increasing (for a tutorial review, see Lee, 2008), the use and application of this approach in the field of visual attention remains limited. In the present study, we examined the time course of facilitation by contextual cueing (Chun \& Jiang, 1998) and its effect on attentional guidance by using a promising new approach that combines a psychometric function with Bayesian hierarchical methods. Psychometric functions have been used in visual attention research to model and predict performance. In particular, the speed-accuracy tradeoff (SAT) function has been utilized to describe the rate of visual information processing as a function of time and how the rate is changed by covertly attending to the visual information (Carrasco \& McElree, 2001; McElree \& Carrasco, 1999). The use of the SAT function in contextual cueing literature is also new, which allowed us to investigate the time course of facilitation more precisely than was previously possible.

In the SAT task, participants engage in a visual search task in which they are required to respond immediately once a sound probe stimulus is presented. At shorter stimulus-onset 
asynchronies (SOAs; e.g., $\sim 40 \mathrm{~ms}$ between the onset of a search display and that of the sound probe), accuracy is at the chance level, whereas at longer SOAs (e.g., > $500 \mathrm{~ms}$ ) performance reaches an asymptote. This growth of accuracy (also termed an "accuracy curve") as a function of SOAs has been described more precisely with the SAT function:

$d^{\prime}(t)=\lambda\left(1-e^{(1-\beta(t-\delta)}\right), t>\delta$, else 0

The parameter $\lambda$ reflects the asymptotic accuracy. The $\beta$ and $\delta$ are pre-asymptotic parameters, which respectively reflect the growth rate of accuracy and the time point when the accuracy leaves the chance level. In the present study, we adopted the probability-modeling approach to evaluate the data acquired in the visual-search experiment. For this purpose, the numbers of correct responses, instead of conventional d', are modelled as a function of the numbers of trials in each of the SOA conditions using the binomial distribution. The SAT function is employed to describe the growth of parameter $\theta$ (i.e., success rate) as a function of individual temporal lengths of the SOAs.

This study further extends this use of the SAT function by examining how contextual cueing facilitates attentional guidance in visual search and how facilitation unfolds with time within a trial. Contextual cueing refers to the phenomenon that a repeated spatial layout in a visual search task leads to a faster reaction to a subsequent target stimulus (Chun \& Jiang, 1998). In a typical contextual-cueing task, participants search for a target stimulus, for example a rotated alphabet $\mathrm{T}$, while ignoring distractor stimulus L. Unbeknownst to the participants, the configuration of distractors (i.e., context) is paired with the target location, and this pairing then is presented repeatedly over time, thereby allowing associative learning of these two sets of information. A common finding is that response time to identify the target shortens with repetitions relative to performance with newly generated contexts. The juxtaposition of performance with repeated versus novel targets is often termed "the contextual-cueing effect." To date, numerous studies have been conducted to examine mechanisms underlying this contextualcueing effect (for reviews, see Goujon, Didierjean, \& Thorpe, 2015, and Sisk, Remington, \& Jiang, 2019). One of the many debates surrounding this effect has focused on that component of visual search that is facilitated by contextual cueing.

The existing literature on this topic has revealed that mainly two components in visual-search processing are facilitated: attentional guidance and response selection (Kunar et al, 2007; Kunar \& Wolfe, 2011). The original study (Chun \& Jiang, 1998) assumed that contextual cueing facilitates search performance by guiding the attentional deployments during search. They provided support for this proposal by showing that the slope of a search function becomes steeper after contextual cues are learned. Sisk et al. (2019) termed this the "early locus" of a contextual-cueing effect. A number of studies have reported evidence for this account with various measures. Peterson and Kramer (2001) recorded eye movements during visual search and showed that fewer fixations were needed to find a target in old contexts than in new contexts, suggesting that the learned contextual cue effectively guided attention to the target position. Event-related potential (ERP) studies also supported the early locus account (Johnson, Woodman, Braun, \& Luck, 2007; Olson, Chun, \& Allison, 2001). A behavioral study from Ogawa, Takeda, and Kumada (2007) offers indirect evidence of facilitated attentional guidance by demonstrating that contextual cueing enables a participant to inhibit distractors, thus leading to more efficient search. Recently, Harris and Remington (2017) provided strong evidence for attentional-guidance facilitation by combining pop-out search with eye-movement measures. Taken together, these previous studies appear to support the notion that contextual cueing expedites the early locus of visual search process.

If attentional guidance is facilitated by contextual cueing, another question yet to be answered is how quickly this facilitation arises. Geyer, Zehetleitner, and Muller (2010) suggested that the expedition occurs during the saliency computation (Muller, Heller, \& Ziegler, 1995). They limited processing time either by masking a display soon (i.e., $90 \mathrm{~ms}$ ) after the stimuli appeared on a screen, or by instructing participants to make a speeded response. The result showed that signal detection accuracy was higher for the old displays than for the new displays, supporting the possibility that an early locus, such as feature signal computation of visual search, is expedited. This is consistent with the ERP activities in V1 and V2 being modulated by learned contexts, suggesting the involvement of facilitation at earlier processes (Olson, Chun, \& Allison, 2001). Chaumon, Drouet, and Tallon-Baudry (2008) have also provided evidence for the early onset of contextual facilitation with MEG (magnetoencephalography). These neurological findings do not necessarily mean that attentional processing per se is facilitated; however, these studies have collectively demonstrated that facilitation by contextual cueing arises early in the time course of visual-search processing.

This study extends previous research by using the probability-modeling approach reviewed below, and as well as connectionist modeling (Brady \& Chun, 2007), both of which have made an important contribution to clarifying the cognitive mechanisms of the observed findings. Thus far, two studies adopted probability modeling to examine the underlying processes of contextual cueing (Sewell, Colagiuri, \& Livesey, 2018; Weigard \& Huang-Pollock, 2014). These studies utilized the diffusion model (Ratcliff, 1978), in which reaction time arises from a probability distribution called the Wiener process. The diffusion model decomposes reaction times into four components, including the responsethreshold parameter $\alpha$ and a rate of information accumulation 
parameter $\mathrm{v}$ (i.e., drift rate). Revealing that these parameters differ between old and new contexts, Sewell et al. (2018) suggested that contextual cueing modulates not only the response threshold, which corresponds to the response facilitation account, but also the rate of information accumulation, which corresponds to the earlier processes of visual search. As the latter parameter does not solely correspond to attentional guidance facilitation, these authors also proposed that facilitation at a more perceptual level also takes part in contextual cueing. In the same vein, Weigard and Huang-Pollock (2014) studied the contextual-cueing effect in children with attentiondeficit hyperactivity disorder (ADHD), who had been considered unable to benefit from contextual cueing. Although it was not reflected in the reaction-time measure, these children's data also showed a lower response threshold in the learned contexts. As such, the probability modeling approach potentially reveals a new perspective that is not apparent only with reaction-time measures.

The present study combined this promising approach with the conventional SAT task. We attempted to decompose the accuracy of visual search into the above-mentioned $\beta, \delta$, and $\lambda$ parameters by using a contextual manipulation. The interpretation of these parameters, however, requires caution because this is a descriptive model. However, it is also possible that contextual cueing has implications for the time course of attentional processing. Geyer et al. (2010) suggested that contextual cueing facilitates search because it increases the saliency of a target item, which suggests the possibility that parameter $\delta$ is influenced by contextual learning, because the strength of attentional guidance might be greater for old than for new contexts, leading to rapid facilitation early in the time course of a search trial. On the other hand, if contextual learning influences the speed of attentional accumulation, this might be reflected in parameter $\beta$. It is also plausible that both mechanisms are effective, and, therefore, both parameters might change as a result of contextual cueing.

As such, if contextual cueing affects the attentional guidance during visual search as many previous studies have suggested, then the pre-asymptotic parameters, $\beta$ and/or $\delta$, should be modulated accordingly. In this way, the SAT task allows us to examine the temporal dynamics of a search, with more focus on data-generation processes with the probability modeling approach; in turn, this would add to the accumulating evidence that attentional guidance is facilitated by contextual cueing. Although the adaptation of accuracy measures and response-signal procedure to the contextual-cueing effect is not new (Chun \& Jiang, 1998, Experiment 5; Geyer et al., 2010), we scrutinized how the facilitation changes as a function of time available for processing visual information by setting multiple conditions of SOAs, ranging from a very short (40 $\mathrm{ms})$ to a long enough period $(2,000 \mathrm{~ms})$, assuming that response accuracy reaches the asymptote by then. By combining this manipulation with the SAT function-based modeling, we were able to describe the time course of the effect without employing complicated experimental designs.

The purpose of the present study was twofold: First, to adopt the Bayesian hierarchical modeling approach that expresses the time course of data-generation processes during visual search by extending the SAT function; and secondly, to characterize the time course of facilitation due to contextual cueing with the constructed model. If the early stages of visual search are expedited, as suggested by the original study of Chun and Jiang (1998) and others, then the pre-asymptotic parameters should be affected. Specifically, if processes prior to target detection, information accumulation in particular, are speeded up, then the growth-rate parameter $\beta$ should be larger for learned contexts than for newly generated contexts. At the same time, we also compared accuracy between context conditions (old vs. new) at each SOA condition. If the quality of perceptual evidence entering a decision process is enhanced in learned contexts, as suggested by the diffusion-modeling study (Sewell et al., 2018), this would mean that quite early processes of visual search are facilitated by contextual cueing. Thus, we predicted that accuracy benefits should appear at shorter SOAs where a limited time is available to process stimuli and only earlier processing of search is completed.

\section{Methods}

\section{Participants}

Nineteen undergraduate students participated in the experiment for a monetary compensation of $¥ 3,500$ (approximately US\$32) or partial fulfillment of a course credit. Among them, three participants failed to meet the criteria described below in a practice session, thus they did not complete subsequent sessions and are not included in the data. All the participants provided written informed consent, and reported that they had normal or corrected-to-normal vision and audition. None of them was aware of the purpose of the study.

\section{Materials}

All experimental sessions were conducted in a dimly-lit room. Experimental stimuli were presented on a 24-in. display (BenQ XL2420T), and the experiments were programmed with PsychoPy (Peirce, 2009). A sound stimulus used in an experimental session was presented through a headphone (SONY MDR-Z1000). Participants were seated at a viewing distance of $65 \mathrm{~cm}$ from the display, and a chin rest was used to ensure the distance was fixed throughout the experiment.

A visual search task in which participants search for a target stimulus among distractor stimuli was used throughout the experiment. In the search task, the alphabet letter T was used as a target and the letter L served as a distractor. The display 
contained one T and $11 \mathrm{Ls}$. The T was rotated $90^{\circ}$ clockwise or anticlockwise, which was determined randomly on every trial. Likewise, each of the L letters was rotated $0^{\circ}, 90^{\circ}, 180^{\circ}$, or $270^{\circ}$. All stimulus items were of the same size, subtending $0.7 \times 0.7^{\circ}$.

Stimuli were presented on three imaginary concentric circles. The diameter of each circle was, respectively, $3.48^{\circ}$, $6.95^{\circ}$, and $10.41^{\circ}$. The $\mathrm{T}$ always appeared on the middle circle, in order to equate a target eccentricity in each trial. Of the rest of the stimuli, three were presented on the inner circle, four were presented on the middle circle, and six were presented on the outer circle. Each circle comprised six, 12, and 18 equidistant possible item locations, respectively, ensuring that crowding would not occur.

Eight old displays were used to form old displays that were uniquely designed for each participant. Eight target locations were selected; these were used in both old and new displays. In the old displays, the set of distractors was always paired with a particular target location. In the new displays, the same target locations were used as in the old displays, but the locations of distractors presented with these targets were determined on a trial-by-trial basis, which meant that any distractor layout could not predict the target location.

\section{Procedure}

First, the participants completed a learning session. In this session they engaged in the visual search task over a series of trials. Each trial started with a 500-ms fixation cross appearing at the center of a screen, which was followed by a search display containing a target and 11 distractors. Participants were asked to search for the target letter among the $\mathrm{L}$ distractors and to identify the orientation of the target (a $\mathrm{T}$ rotated $90^{\circ}$ clockwise or anticlockwise). They were instructed to respond by pressing a key as quickly and accurately as possible. Unlike the SAT procedure described below, the search display remained on the screen until a response was made, and no time limit for the response was imposed. The participants completed 24 blocks of eight trials, and each display was presented once in a block. Unbeknownst to them, newly generated displays were presented during the final four blocks in order to test whether visual searches for the old displays were indeed facilitated.

The completion of the learning session was followed by a practice session of the SAT task. The procedure is presented in Fig. 1. After a fixation cross was shown for $750 \mathrm{~ms}$, participants were presented with a display that consisted of the same stimuli as in the learning session, and searched for the target among distractors. The display presentation was followed by a sound-probe stimulus, which forced the participants to make a response immediately even when they had not found the target. The participants were instructed to respond within $400 \mathrm{~ms}$ after the sound probe, otherwise an error feedback was presented on the screen. The SOAs had eight conditions, which

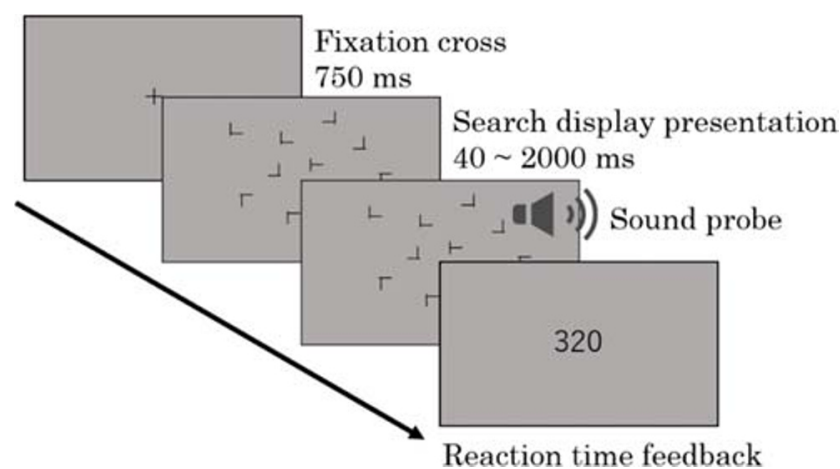

Fig. 1 A schematic representation of a trial sequence in the speedaccuracy tradeoff (SAT) task

were $40,90,150,200,300,500,900$, and 2,000 ms, respectively. These SOAs were presented in random sequences. The search display remained on the screen until response or $400 \mathrm{~ms}$ after the sound probe when no response was made in the specified time. When participants responded within 400 $\mathrm{ms}$, a reaction time feedback was given. No accuracy feedback was given after the response. The practice session started with a search display that contained only the target stimulus, where participants were told to identify the orientation of a target. This procedure was employed to allow participants to become accustomed to making responses in the specified time period, which is not a common experimental task. When accuracy in trials with each set size (i.e., the number of stimuli on a display) reached $60 \%$, a distractor was added to the display. Regardless of accuracy, participants completed at least 20 trials with each set size. Thereafter, accuracy was calculated at the end of every trial as a ratio of a number of correct trials to the number of all trials completed at the current set size. The configuration of the search display was randomly generated for each trial. This sequence was repeated until the number of stimuli on the display equaled 12 . If the number of stimuli on the screen did not reach this criterion $40 \mathrm{~min}$ after the beginning of the practice session, the participants did not conduct a session that followed.

Finally, a test session of the SAT task was conducted. Unlike the practice session, the set size was 12 from the beginning and did not change as the task progressed. Participants completed two blocks of the SAT task. One block presented participants with the displays learned in the learning session; the other block contained newly generated displays. Each block consisted of 1,120 trials. The order of these two blocks was counterbalanced across participants. The whole experiment took about $4 \mathrm{~h}$, and the participants were given a 45 min break in the middle of the experimental sessions.

\section{Analysis}

The data were analyzed using free statistic software $\mathrm{R}$ (version 3.5.1; 2018-07-02), and $\mathrm{R}$ packages including MASS 
(Venables \& Ripley, 2002), lmerTest (Kuznetsova, Brockhoff, \& Christensen, 2017), and lme4 (Bates, Maechler, Bolker, \& Walker, 2015) were used for a priori contrast coding, the linear mixed model, and the generalized linear mixed model, respectively. The learning session involved a 2 (context; old or new) $\times 6$ (epochs; four blocks were combined into one epoch in order to increase the power, such that the session involved six epochs) within-subject design. Repeated contrasts were coded to reflect six epochs, and the reaction-time data were analyzed with the linear mixed model, with participants as a random intercept. We focused mainly upon the last two epochs because we considered the fifth epoch to be when the context learning was completed, and the sixth epoch to be when pairings between a distractor and target locations were destroyed and learned information could not be used to facilitate a search.

In order to examine whether repeating displays facilitate search guidance, an analysis of accuracy on each condition of the SAT test session with the generalized linear mixed model was conducted, with participants treated as a random intercept. Sum contrasts were coded, with the old condition as +0.5 and the new condition as -0.5 , which enabled us to compare the accuracies of these conditions on each SAT condition without the need for multiple comparisons; thus only one degree of freedom was spent.

We estimated the pre-asymptotic parameters $\beta$ and $\delta$ of the SAT function by modeling accuracy in the SAT test session in a Bayesian, hierarchical manner. Models were constructed and computed using the Stan and RStan package (Stan Development Team, 2019), the R interface to Stan. In our model, we assumed that responses were made based on the binomial distribution. The number of hits per participants in each condition is represented as:

$\alpha_{i, k} \sim \operatorname{Binomial}\left(n_{i},{ }_{k}, \theta_{i, k}\right)$

Here, $\mathrm{n}$ is a number of trials and $\theta$ describes a hit rate. The subscripts $\mathrm{i}$ and $\mathrm{k}$ refer, respectively, to each participant and context condition (two levels - old or new). The assumption here is that responses are independent of each other. Then, we assumed that this probability distribution of responses, $\theta$, changes as a function of SOAs (described as $t$ in the formula), as follows:

$\theta_{i},{ }_{j}(t)=0.5+\lambda\left(1-\exp \left(1-\beta_{i, k}\left(t-\delta_{i, k}\right)\right)\right)$

This model is derived from conventional SAT functions. Hence $\lambda$ is an asymptotic parameter, whereas $\beta$ and $\delta$ are the pre-asymptotic parameters that describe a portion of the function before $\theta$ reaches an asymptote; 0.5 was added to the conventional SAT function in order for $\delta$ to be a parameter reflecting the time point when $\theta$ leaves the chance level, $50 \%$. In this model $\lambda$ is first estimated with the prior $\operatorname{Normal}(0,0.5)$, and then transformed with the inverse logit function to values within $(0,0.5)$. This procedure was employed to ensure that the theoretical maximum of $\theta$ would be 1 . Otherwise, a model with a free $\lambda$ failed to converge, either when more weak priors (e.g., Normal $(0,1))$ were used or when the transformation with the inverse logit function was not employed.

To investigate whether there were any differences in parameter values between old and new displays, we calculated Bayes factors for comparing each parameter of the full model with a model in which the parameter $\beta$ varied between the display conditions with the null model. The calculation was conducted by means of bridge sampling with the bridgesampling package (Gronau, Singmann, \& Wagenmakers, 2017) on R. We found that there is strong evidence in favor of a model over the null when a Bayes factor exceeds 100, according to Jeffreys' criteria (1961; as cited in Lee \& Wagenmakers, 2013).

The parameters in models were estimated by generating a Markov-Chain Monte Carlo (MCMC) sample from posterior distributions. Weakly informative prior distributions, usually with standard deviations of 10 , were employed as the prior distributions for each parameter. For the standard deviations of each parameter's values, we used the Cauchy distribution with a standard deviation 2.5. In order for the estimates to be stable, we used 12,000 steps of four MCMC chains, among which 2,000 steps were discarded as a warm-up. We concluded that chains converged successfully when $\hat{R}$ values were under 1.04 .

\section{Results}

\section{Reaction time in the learning session}

To determine whether contextual information was successfully learned, reaction-time data in the learning session (Fig. 2) were analyzed with the linear mixed model. A priori contrast was coded to compare reaction time between each epoch; see Table 1 for results. Reaction time in epoch 6, where the association between distractors and a target was not retained, was longer than in epoch 5 ( $b=152.52, S E=27.19, t=5.61, p<$ $.01)$. This indicates that contextual cueing was acquired by epoch 5, and it also suggests the learned association was weakened by the changes in epoch 6 , which shows longer reaction times.

\section{Speed-accuracy tradeoff (SAT) accuracy}

The accuracy in the SAT test session (Fig. 3) was analyzed with the generalized linear mixed model. A priori contrast was coded to compare accuracy between context conditions (old vs. new) in each SOA condition. As shown in Table 2, correct responses in the old-context condition were more likely to occur than in the new-context conditions in SOA conditions 


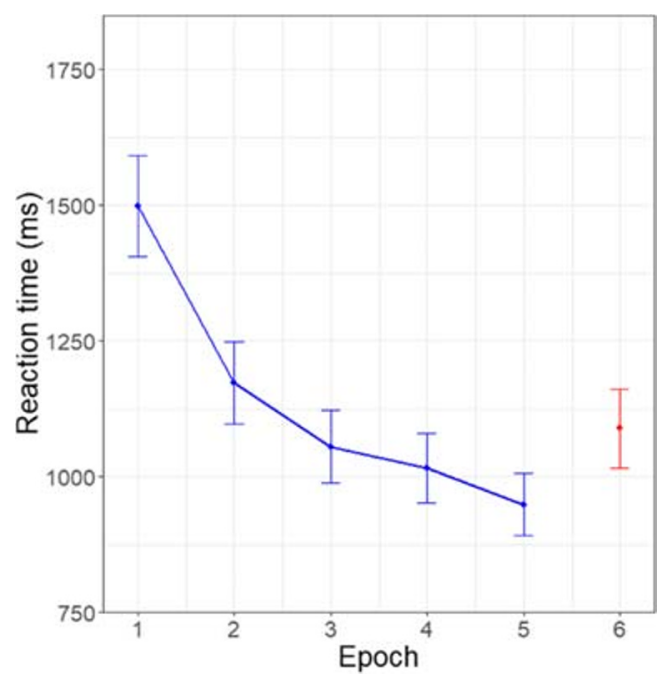

Fig. 2 Mean response time in the learning session. The error bars represent the standard error values

of $90,150,200,300,500$, and $900 \mathrm{~ms}(p s<.01)$. This result shows that participants can respond to the learned contexts even when only a small amount of time is available to process a search display, suggesting that the benefit appeared quite early in the process of visual search.

At the longest SOA (i.e., 2,000 ms), no difference in accuracy was observed between the display conditions. This result may be attributed to a ceiling effect, namely the fact that accuracy was quite high in both conditions (>97\%). Furthermore, the results from the learning session showed that most searches were completed within $2,000 \mathrm{~ms}$. In the standard contextual cueing paradigm without any time limitation, no accuracy difference was observed. Our results are in line with those of previous studies.

The reaction-time distributions in the SAT task are shown in Fig. 4. The distribution was plotted as a stack histogram, in which each participant was represented by a different color. We used a linear mixed model to investigate the interaction between the SOA and display conditions. The SOA, display conditions, and correct/incorrect trials were included as fixed effects, and participants as a random intercept. Participants

Table 1. Results of the analysis on reaction time data in the learning session

\begin{tabular}{llllll}
\hline & $b$ & $S E$ & $t$ & $p$ \\
\hline (Intercept) & & 1126.8 & 60.64 & 18.58 & $<.01$ \\
Epoch & $2-1$ & -291.14 & 27.19 & -10.71 & $<.01$ \\
& $3-2$ & -116.71 & 27.19 & -4.29 & $<.01$ \\
& $4-3$ & -45.35 & 27.19 & -1.67 & .10 \\
& $5-4$ & -72.24 & 27.19 & -2.66 & $\mathbf{. 0 1}$ \\
& $6-5$ & 152.52 & 27.19 & 5.61 & $<.01$ \\
\hline
\end{tabular}

Significant values $(\mathrm{p}<.05)$ are shown in bold

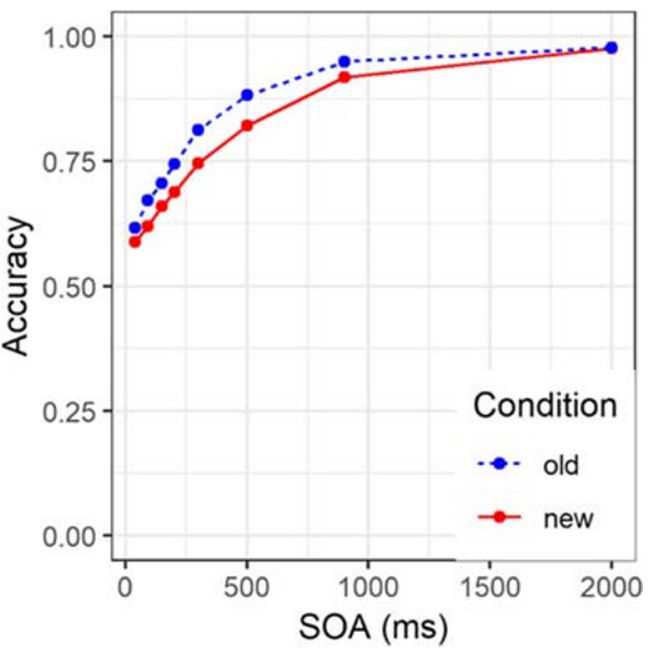

Fig. 3 Mean accuracy in the speed-accuracy tradeoff (SAT) test session. The blue dotted line represents the old condition, and the red solid line represents the new condition

responded faster as the SOA became longer, which is reflected as a main effect of SOA $(b=-0.02, S E=0.003, t=-5.36, p<$ $.01)$. Correct trials and old-context trials yielded shorter reaction times $(b=-14.56, S E=1.66, t=-8.75, p<.01 ; b=-11.79$, $S E=2.10, t=-5.62, p<.01)$. The two-way interactions between the correct/incorrect and display conditions were statistically significant $(b=-4.96, S E=2.42, t=-2.06, p=.03$ ). This interaction is shown in Fig. 5, in which the reaction time was plotted with an interactions package (Long, 2019). This plot reveals a larger difference between new and old display

Table 2. Results of the analysis on accuracy data in the speed-accuracy tradeoff (SAT) test session

\begin{tabular}{llllll}
\hline & & $\mathrm{b}$ & $\mathrm{SE}$ & $\mathrm{t}$ & $\mathrm{p}$ \\
\hline (Intercept) & & 1.55 & 0.11 & 14.44 & $<.01$ \\
SOA & $90-40$ & 0.20 & 0.05 & 3.69 & $<.01$ \\
& $150-90$ & 0.18 & 0.05 & 3.26 & $<.01$ \\
& $200-150$ & 0.17 & 0.05 & 3.04 & $<.01$ \\
& $300-200$ & 0.36 & 0.06 & 6.18 & $<.01$ \\
& $500-300$ & 0.52 & 0.07 & 7.81 & $<.01$ \\
& $900-500$ & 0.91 & 0.09 & 10.49 & $<.01$ \\
SOA40 & cono-900 & 1.08 & 0.13 & 8.08 & $<.01$ \\
SOA90 & conditions & 0.10 & 0.08 & 1.31 & .19 \\
SOA150 & conditions & 0.21 & 0.08 & 2.81 & $<.01$ \\
SOA200 & conditions & 0.21 & 0.08 & 2.77 & $\mathbf{. 0 1}$ \\
SOA300 & conditions & 0.38 & 0.09 & 4.46 & $<.01$ \\
SOA500 & conditions & 0.48 & 0.10 & 4.76 & $<.01$ \\
SOA900 & conditions & 0.50 & 0.14 & 3.50 & $<.01$ \\
SOA2000 & conditions & 0.00 & 0.23 & 0.00 & .99 \\
\hline
\end{tabular}

Significant values $(\mathrm{p}<.05)$ are shown in bold

$S O A$ stimulus-onset asynchrony 


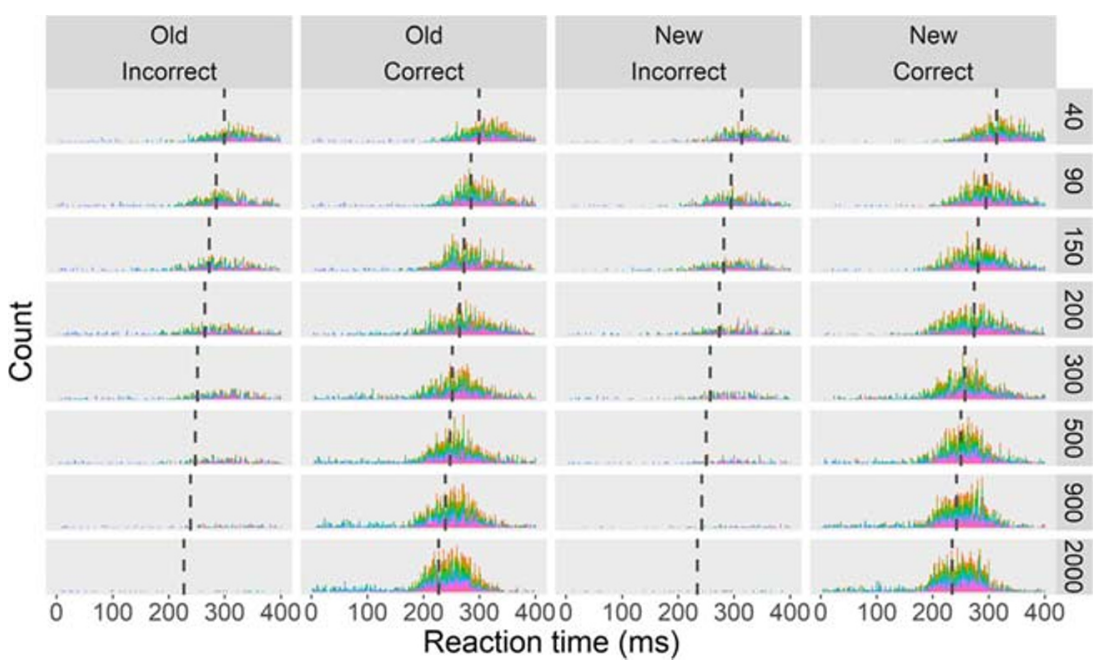

Fig. 4 The reaction-time distributions in the speed-accuracy tradeoff (SAT) test session plotted as a stack histogram. Different colors represent the distribution of each participant

conditions in the incorrect trials compared to the correct trials. Correct/incorrect and SOA conditions, and display and SOA conditions were not statistically significant, nor was the threeway interaction between correct/incorrect, display, and SOA conditions $(b=-0.01, S E=0.004, t=-1.30, p=.19 ; b=0.01$, $S E=0.01, t=1.44, p=.15 ; b=-0.01, S E=0.01, t=-1.77, p=$ $.07)$.

These results suggest that participants could use additional time after the response signal to search before the response execution, but the amount of time that could be used was not very long. By taking account of the fact that accuracy reached $100 \%$ at a SOA of 2,000 ms, the search should be completed by then. Therefore, we infer that the reaction time in this condition was made up of motor or response-related components. Considering that the longest time needed for a response was approximately $299 \mathrm{~ms}$ (the 40-ms SOA condition) and the shortest was $227 \mathrm{~ms}(2,000 \mathrm{~ms})$, the implication is that an additional $72 \mathrm{~ms}$ can be used for search until the responserelated processes set in.

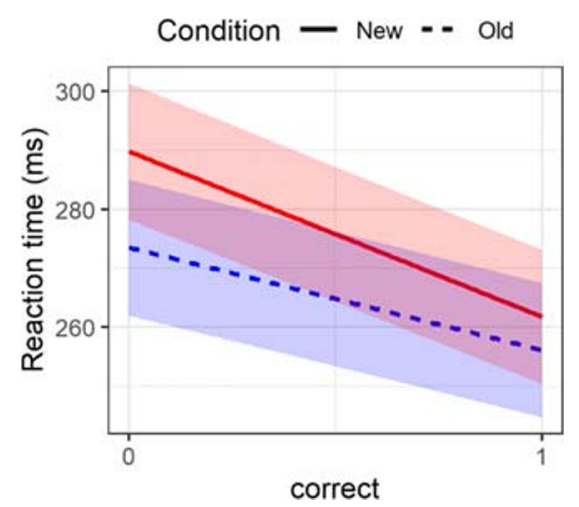

Fig. 5 Schematic representation of the interaction between the display condition and correct/incorrect trials. The blue dotted line represents the old condition, and the red solid line represents the new condition

\section{Modeling}

In order to examine changes in pre-asymptotic parameters $\beta$ and $\delta$ as a function of context conditions, we constructed four models: (1) a model in which both $\beta$ and $\delta$ are variable across the two context conditions (full model); (2) a model in which only $\beta$ varies across the conditions ( $2 \beta$ model); (3) a model in which only $\delta$ varies across the conditions ( $2 \delta$ model); and (4) a model in which neither of these two parameters varies across the conditions (null model). Prior to comparisons between the models described above and the null model with Bayes factors, which are ratios of log marginal likelihoods of two models, negative log marginal likelihoods (i.e., free energies) for each model were calculated. The free energy of the null model (784.37) was the largest among the three models, followed by the full model (748.03), and then by the $2 \beta$ model (718.06). The calculation of the negative log marginal likelihood with the bridge sampling method did not converge for the $2 \delta$ model.

In order to observe the fit of the $2 \delta$ model compared with other models, we simulated accuracy as a function of SOAs with the estimated parameter values of the $2 \delta$ model and the $2 \beta$ model. The estimation is plotted in Fig. 6 . When this estimate is compared to the actual result in Fig. 3 , the $2 \beta$ model predicted the accuracy better than the $2 \delta$ model. In addition, the fit did not improve when variable $\delta$ s were added to the model, as was observed in the comparison between the full model and the $2 \beta$ model ( 748.03 vs. 718.06 ; for a comparison with the Bayes factor, see Table 2). These two examinations showed that the $2 \delta$ model may not fit the data better than either the $2 \beta$ model or the full model, although its fit may still be better than the null model.

The implication of the above discussion is that the $2 \beta$ model may fit better with the data than the null and full model. 

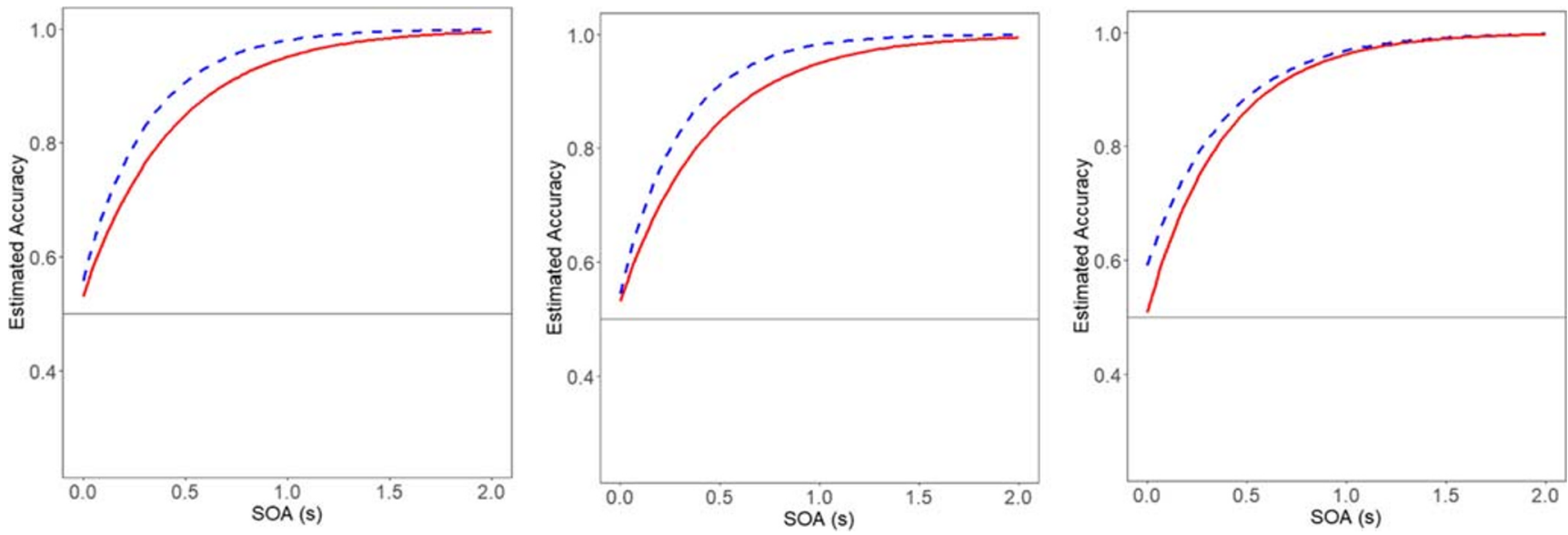

Fig. 6 The accuracy estimated by the full model (left panel), the $2 \beta$ model (middle panel), and the $2 \delta$ model (right panel). The blue dotted lines represent the old condition, and the red solid lines represent the new condition

In order to confirm this implication, we calculated Bayes factors to compare (1) the full model versus the null model; (2) the $2 \beta$ model versus the null model; and (3) the full model versus the $2 \beta$ model. Each Bayes factor is presented in Table 3. These data provide very strong evidence in favor of the full model $\left(\mathrm{BF}>10^{11}\right)$; also, evidence in favor of the $2 \beta$ model over the null model was extremely strong $\left(\mathrm{BF}=10^{24}\right)$. Comparison between the $2 \beta$ model and the full model yields a Bayes factor of over $10^{13}$, which provides very strong evidence according to the criteria determined by Jeffreys (1961); that is, the comparison shows that the $2 \beta$ model fits better with the current data than does the full model. Overall, the analysis with Bayes factors demonstrated that the $2 \beta$ model fits well with the data obtained in this study. Thus, the present analysis showed that the data obtained in this experiment could be described by varying the growth rate parameter $\beta$ between the display conditions, with the starting point parameter $\delta$ fixed.

\section{Summary of the results}

Overall, these analyses provide support for the early locus of a contextual-cueing effect. The analysis of accuracies in the SAT task demonstrates that the contextual cueing benefit appeared very early in the time course of visual search. The result that responses were more accurate in the old than in the new condition even when SOAs were quite short cannot be explained solely in terms of the response facilitation account. The Bayesian hierarchical modeling approach enables us to examine how the growth of accuracy, as a function of the SOAs, varies depending on whether contexts are learned or not. We concluded that the early facilitation of search was involved in the contextual cueing benefit because the preasymptotic parameter $\beta$ differed between the context conditions. Of course, this is merely a speculation from these parameter estimations; some caution is warranted in interpreting this finding.

\section{Discussion}

We explored a method for examining the time course of facilitation resulting from contextual cueing by characterizing how early attentional guidance is facilitated. Our secondary goal was to adopt a Bayesian hierarchical modeling approach with the SAT function, which is a conventional psychometric function used to describe the rate of information processing. The experiment with the SAT task and the Bayesian hierarchical modeling approach revealed that the model captured the time course of visual search well: contextual cueing benefits are reflected on the earlier processing of visual search. Specifically, more accurate responses emerged only when a limited amount of time was available to process search displays. This result was reflected in the higher growth rate of

Table 3. Results of the Bayesian hierarchical modeling of accuracy growth in the speed-accuracy tradeoff (SAT) test session

\begin{tabular}{llll}
\hline Log negative marginal likelihoods of each model & Bayes factor \\
\hline Full model & 748.03 & (1) Full model vs. null model & $4.20 * 10^{11}$ \\
$2 \beta$ model & 718.06 & (2) $2 \beta$ model vs. null model & $4.34 * 10^{24}$ \\
Null model & 774.79 & (3) $2 \beta$ model vs. Full model & $1.03 * 10^{13}$ \\
\hline
\end{tabular}


accuracy as a function of the SOAs, such that information accrual during a search was more rapid as a result of contextual cueing. These results clearly show that facilitation by contextual cueing appears early in the time course of visualsearch processing, adding to evidence that demonstrates attentional guidance facilitation by contextual cueing (Chaumon et al., 2008; Chun \& Jiang, 1998; Johnson et al., 2004; Olson et al., 2001).

With a Bayesian hierarchical modeling approach and the SOA manipulation in the SAT procedure, we have provided additional evidence showing how accuracy grows as a function of time in a trial, and how its growth changes as a result of contextual cueing. The potential contributions to the field are twofold. First, the applications of the present study's approach to questions concerning different cognitive processes can be easily adopted, and second, this approach allows a continuous and quantitative description of the time course of T-among-L search after contextual learning.

Our approach combining a psychophysical function with Bayesian hierarchical modeling can be applied not only to performance in SAT-like procedures but also to other cognitive processes. In our modeling approach, we posit that the accuracy of target identification becomes more available as processing time increases, and that its growth rate depends on the learning of contextual cueing. Whereas conventional experimental designs allow us to test the existence of relationships between independent and dependent variables, modeling enables us to examine the details of the relationships by describing the data as a mathematical function. This, in turn, makes it possible to predict the changes in output of the model (accuracy in the present study) as a result of a variation of input (in the current example, available processing time or the presence of contextual cues). This type of modeling further facilitates the testing of relevant predictions that potentially update and improve the model. Moreover, such modeling may ultimately lead to a deeper understanding of mechanisms underlying a given model. As far as the phenomenon of interest can be described as a relationship between the input and output of a function (which should be possible in many cognitive psychology paradigms), this approach can be easily applied. As Logan (1988) observed, task facilitation by practice can be described as a power function: the application of this to the probability model and Bayesian hierarchical modeling would, for example, allow us to examine individual differences in the practice effect or in factors that influence the practice rate in a quantitative way.

In the present study, our approach enabled a quantitative description and the evaluation of a time course. How performance improves as processing time increases (i.e., a relationship between the input and output) has not been well studied, although earlier processing facilitation by contextual cueing (i.e., the presence of a relationship) is well documented. Previous studies have demonstrated that participants can perform well even under conditions in which only a brief time is available for processing displays after learning contexts. Chun and Jiang (1998) presented participants with briefly flashed displays and requested that participants search for a target among distractors. Their study showed that such learned displays produced more accurate performance than new displays. It has been shown that more responses with short reaction times were made when participants were presented with repeated displays than with new displays (Johnson et al., 2004). By providing evidence of how accuracy grows as a function of time available for processing displays, our study further corroborates the view that earlier processing of visual search temporal dynamics are facilitated by contextual cueing (Chaumon et al., 2008; Johnson et al., 2007; Olson et al., 2001), as well as behavioral measures (Chun \& Jiang, 1998; Geyer et al., 2010).

The estimates of the parameters in the SAT function support the earlier locus account. Studies in visual attention have utilized the SAT procedures to investigate the speed of information processing, instead of the conventional reaction-time measures that fail to provide information that reveals whether or not information accrual is speeded (Carrasco \& McElree, 2001; McElree \& Carrasco, 1999). Based on the function used in the SAT procedure, we modelled responses in the SAT task and observed that the parameter $\beta$, which reflects the rate of information processing, varies depending on contextual cueing. The finding that the speed of information processing increases following learning contexts fits well with the studies supporting the early locus account. Several studies with various measures have demonstrated that processing before an overt response becomes efficient as a result of contextual cueing (Harris \& Remington, 2017; Johnson et al., 2004; Ogawa et al., 2007; Peterson \& Kramer, 2001). Future studies may address this issue by employing more difficult search displays to obtain below-chance performance and to observe whether these starting points do indeed differ between the two display conditions. Limiting the display presentation duration is one method, although it might not be an effective method of obtaining below-chance performance, considering that a brief presentation of $200 \mathrm{~ms}$ in Chun and Jiang's study (1998; Experiment 6) led to an accuracy above chance. It might be more plausible to use the similarity to a target of a distractor, which increases the reaction time but does not affect the magnitude of contextual cueing (Jiang \& Sisk, 2019). Overall, however, these estimations showed that the contextual cueing benefits appear at the early attentional guidance during search.

Another aspect of these data concerns the consistency evident in the diffusion model estimates and the new hypothesis based on it, as provided by Sewell et al. (2018). Based on their finding that one of the diffusion model parameters, the drift rate $\mathrm{v}$, was larger in old than in new contexts, they proposed a hypothesis that perceptual templates of repeated displays were 
formed to increase the quality of information that drives the decision-making process in visual search. A result from the present study adds to the above proposal in suggesting that facilitation by contextual cueing occurs largely from earlier stages of the search process. To be specific, responses were more accurate in repeated contexts than in new contexts, even when a limited amount of time was available to process the display information. By relating our results to the finding of Sewell et al. (2018), it can be speculated that the quality of accumulated information is higher for repeated than for new displays, leading to more accurate responses in learned displays.

However, we note that interpreting the parameters in regard to the SAT function and mapping them to underlying psychological processes is not simple. Although the accuracy growth parameter in exponential models can be taken as information accrual speed, changes in this parameter can be confounded with the decision threshold (Ratcliff, 2006). Matzke and Wagenmakers (2009) also cautioned against the interpretation of a parameter in descriptive models, and recommended treating it as a mere description without relating it to psychological processes. Therefore, it is necessary to acknowledge that our model is not a cognitive model that reflects the mechanisms behind the observation, but rather a descriptive model of the observed data.

Although the current study lends support to the presence of an early locus of contextual-cueing effect, these results should not be interpreted as ruling out the possibility that response facilitation also occurs. It is also possible that these two accounts are independent of each other. A limitation of this study is that our model does not include any parameter that corresponds to the response threshold. It was not possible to assess whether a response threshold changes depending on contextual cueing from this study. Further research should be undertaken to distinguish the two accounts of contextual cueing, namely the early-focus and response-facilitation accounts. If both response and attentional guidance facilitation are at work, we would expect that stimulus properties, task goals, or the extent of learning may affect the role each mechanism plays. We suggest that further modeling work is needed to find a model that reflects both mechanisms of contextual cueing benefits. In spite of this limitation, our study offers the potential of a Bayesian modeling approach in this highly studied field, by suggesting an alternative way to describe the observed data as the output of a model, which is in turn determined by the input. This method sheds light on the important role the data descriptions can play, and how the prediction from the model in addition to the conventional and critical experimental designs can further our understanding of cognitive processes.

\section{Conclusion}

The present study was designed to construct, using Bayesian hierarchical modeling, a descriptive model of the time course of visual search and its facilitation by contextual cueing. The experiment relied upon the SAT task. This revealed that early processes that anticipate the locus of a target stimulus are facilitated in learned contexts, which were expressed as a change in the parameter that describes the growth rate of accuracy. Overall, the study strengthens the claim of an early locus account of contextual cueing, although this does not rule out the possibility of facilitation after the spatial locus of a target is found, and several limitations should be noted concerning the interpretations of parameter values. This study is one of the first attempts to apply a Bayesian hierarchical modeling approach to the widely studied issues of attentional guidance in visual search, laying the groundwork for future research in this area.

Funding information This work was supported by JSPS KAKENHI Grant Number JP18K03191.

Data Availability None of the data or materials for the experiments reported here are available, and none of the experiments was preregistered.

\section{References}

Bates, D., Meacher, M., Bolker, B., \& Walker, S. (2015). Fitting Linear Mixed-Effects Models Using lme4. Journal of Statistical Software, $67,1-48$.

Brady, T. F., \& Chun, M. M. (2007). Spatial constraints on learning in visual search: Modeling contextual cuing. Journal of Experimental Psychology: Human Perception and Performance, 33, 798-815.

Carrasco, M., \& McElree, B. (2001). Covert attention accelerates the rate of visual information processing. Proceedings of the National Academy of Sciences of the United States of America, 98, 53635367.

Chaumon, M., Drouet, V., \& Tallon-Baudry, C. (2008). Unconscious associative memory affects visual processing before $100 \mathrm{~ms}$. Journal of Vision, 8, 10-10.

Chun, M. M., \& Jiang, Y. (1998). Contextual cueing: Implicit learning and memory of visual context guides spatial attention. Cognitive Psychology, 36, 28-71.

Geyer, T., Zehetleitner, M., \& Müller, H. J. (2010). Contextual cueing of pop-out visual search: When context guides the deployment of attention. Journal of Vision, 10, 1-11.

Goujon, A., Didierjean, A., \& Thorpe, S. (2015). Investigating implicit statistical learning mechanisms through contextual cueing. Trends in Cognitive Sciences, 19, 524-533.

Gronau, Q. F., Singmann, H., \& Wagenmakers, E.-J. (2017). bridgesampling: An R package for estimating normalizing constants. arXiv Preprint, arxiv:1710.08162

Harris, A. M., \& Remington, R. W. (2017). Contextual cueing improves attentional guidance, even when guidance is supposedly optimal. Journal of Experimental Psychology: Human Perception and Performance, 43, 926-940.

Jeffreys, H. (1961). Theory of Probability. Oxford, UK: Oxford University Press. 
Jiang YV, Sisk CA (2019). Contextual cueing. In Stefan Pollmann (Eds.), Springer Neuromethods: Spatial learning and attention guidance.

Johnson, J. S., Woodman, G. F., Braun, E., \& Luck, S. J. (2004). Implicit memory influences the allocation of attention in visual cortex. Psychonomic Bulletin \& Review, 14, 834-839.

Johnson, J. S., Woodman, G. F., Braun, E., \& Luck, S. J. (2007) Implicit memory influences the allocation of attention in visual cortex. Psychonomic Bulletin \&Review, 14, 834-839.

Kunar, M. A., Flusberg, S., Horowitz, T. S., \&Wolfe, J. M. (2007). Does contextual cuing guide the deployment of attention? Journal of Experimental Psychology: Human Perception and Performance, 33, 816-828.

Kunar, M. A., \& Wolfe, J. M. (2011). Target absent trials in configural contextual cuing. Attention, Perception, \& Psychophysics, 73, $2077-$ 2091.

Kuznetsova, A., Brockhoff, P. B., \& Christensen, R. H. B. (2017). lmerTest Package: Tests in Linear Mixed Effects Models. Journal of Statistical Software, 82, 1-26.

Lee, M.D. (2008). Three case studies in the Bayesian analysis of cognitive models. Psychonomic Bulletin \& Review, 15, 1-15.

Lee, M. D., \& Wagenmakers, E. J. (2013). Bayesian cognitive modeling: A practical course. Cambridge, UK: Cambridge University Press

Logan, G. D. (1988). Toward an instance theory of automatization. Psychological Review, 95, 492-527.

Long, J. A. (2019). Interactions: Comprehensive, User-Friendly Toolkit for Probing Interactions. R package version 1.1.0, https://cran.rproject.org/package $=$ interactions.

Matzke, D. \& Wagenmakers, E. J. (2009). Psychological interpretation of ex-Gaussian and shifted Wald parameters: A diffusion model analysis. Psychonomic Bulletin \& Review, 16, 798-817.

McElree, B., \& Carrasco, M. (1999). The temporal dynamics of visual search: Evidence for parallel processing in feature and conjunction searches. Journal of Experimental Psychology: Human Perception and Performance, 25, 1517-1539.

Müller, H. J., Heller, D., \& Ziegler, J. (1995). Visual search for singleton feature targets within and across feature dimensions. Perception \& Psychophysics, 57, 1-17.
Ogawa, H., Takeda, Y., \& Kumada, T. (2007). Probing attentional modulation of contextual cueing. Visual Cognition, 15, 276-289.

Olson, I. R., Chun, M. M., \& Allison, T. (2001). Contextual guidance of attention: Human intracranial event-related potential evidence for feedback modulation in anatomically early temporally late stages of visual processing. Brain: A Journal of Neurology, 124, 14171425.

Peirce, J. W. (2009). Generating stimuli for neuroscience using PsychoPy. Frontiers in Neuroinformatics, 2, 1-8.

Peterson, M. S., \& Kramer, A. F. (2001). Attentional guidance of the eyes by contextual information and abrupt onsets. Perception \& Psychophysics, 63, 1239-1249.

Ratcliff, R. (1978). A theory of memory retrieval. Psychological Review, $85,59-108$.

Ratcliff, R. (2006). Modeling response signal and response time data. Cognitive Psychology, 53, 195-237.

Sewell, D. K., Colagiuri, B., \& Livesey, E. J. (2018). Response time modeling reveals multiple contextual cuing mechanisms. Psychonomic Bulletin \& Review, 25, 1644-1665.

Sisk, C. A., Remington, R. W., \& Jiang, Y. (2019). Mechanisms of contextual cueing: A tutorial review. Attention, Perception, \& Psychophysics, 81, 2571-2589.

Stan Development Team (2019). RStan: the R interface to Stan. R package version 2.19.2.

van de Schoot, R., Winter, S. D., Ryan, O., Zondervan-Zwijnenburg, M., \& Depaoli, S. (2017). A systematic review of Bayesian articles in psychology: The last 25 years. Psychological Methods, 22, 217239.

Venables, W. N., \& Ripley, B. D. (2002). Modern Applied Statistics with $S$. Fourth edition. Springer.

Weigard, A., \& Huang-Pollock, C. (2014). A diffusion modeling approach to understanding contextual-cueing effects in children with ADHD. Journal of Child Psychology and Psychiatry, 55, 13361344.

Publisher's note Springer Nature remains neutral with regard to jurisdictional claims in published maps and institutional affiliations. 